\title{
FREE CONVECTION FLOW OF CONDUCTING MICROPOLAR FLUID WITH THERMAL RELAXATION INCLUDING HEAT SOURCES
}

MAGDY A. EZZAT

Received 24 March 2004 and in revised form 2 May 2004

The present work is concerned with unsteady free convection flow of an incompressible electrically conducting micropolar fluid, bounded by an infinite vertical plane surface of constant temperature. A uniform magnetic field acts perpendicularly to the plane. The state space technique is adopted for the one-dimensional problems including heat sources with one relaxation time. The resulting formulation is applied to a problem for the whole space with a plane distribution of heat sources. The reflection method together with the solution obtained for the whole space is applied to a semispace problem with a plane distribution of heat sources located inside the fluid. The inversion of the Laplace transforms is carried out using a numerical approach. Numerical results for the temperature, the velocity, and the angular velocity distributions are given and illustrated graphically for the problems considered.

\section{Introduction}

Because of the increasing importance of materials flow in industrial processing and elsewhere, and the fact that shear behavior cannot be characterized by Newtonian relationships, a new stage in the evaluation of fluid-dynamic theory is in progress. Eringen [3] proposed a theory of micropolar fluids taking into account the inertial characteristics of the substructure particles, which are allowed to undergo rotation.

The concept of micropolar fluids deals with a class of fluids that exhibit certain microscopic effects arising from the local structure and micromotions of the fluid elements. These fluids contain dilute suspensions of rigid macromolecules with individual motions that support stress and body moments and are influenced by spin inertia. The theory of micropolar fluids and its extension to thermomicropolar fluids [4] may form suitable non-Newtonian fluid models that can be used to analyze the behavior of exotic lubricants, colloidal suspensions, polymeric fluids, liquid crystals, human and animal blood, and so forth.

Through a review of the subject of micropolar fluid mechanics and its applications, Peddieson and McNitt [19] derived the boundary-layer equations for a micropolar fluid, 
which are important in a number of technical processes, and applied these equations to the problems of steady stagnation point flow, steady flow past a semi-infinite flat plate. Ahmadi [1] studied the fluid flow characteristics of the boundary-layer flow of a micropolar fluid over a semi-infinite plate, using a Runge-Kutta shooting method with Newtonian iteration. The boundary-layer flow on continuous surfaces is an important type of flow occurring in a number of technical processes. Flow in the boundary layer on a continuous semi-infinite sheet moving steadily through an otherwise quiescent fluid environment was first studied theoretically by Sakiadis [20]. Hassanien and Gorla [13] studied the mixed convection in stagnation flow of micropolar fluid over a vertical surface with variable surface temperature and uniform surface heat flux. Bhargava and Rani [2] discussed the heat transfer in a micropolar fluid near a stagnation point. Ezzat and Othman [10] studied the effect of a vertical AC electric field on the onset of convective instability in a dielectric micropolar fluid layer heated from below. Gorla et al. [12] analyzed the heat transfer characteristics of a micropolar fluid over a flat plate. Ezzat et al. [11] studied some problems of micropolar magnetohydrodynamic boundary-layer flow.

The aim of this paper is firstly constructing a mathematical model of boundary-layer equations for conducting micropolar fluid in the presence of heat sources with thermal relaxation time, and secondly studying the effects of some parameters on such fluid.

The solution is obtained using a state space approach [6]. In this approach, the governing equations are written in matrix form using a state vector that consists of the Laplace transforms in time of the temperature, the induced electric field, the microrotation component, and their gradients. Their integration, subjected to zero initial conditions, is carried out means of matrix exponential method. Influence functions in the Laplace transform domain are explicitly developed.

The resulting formulation is applied to a problem for the whole space with a plane distribution of heat sources. The solutions obtained are utilized in combination with the method of images to obtain the solution for a problem with heat sources distributed on a plane situated inside a semispace the surface of which is bounded by an infinite vertical plate. The inversion of the Laplace transform is carried out using a numerical technique [15].

\section{Formulation of the problem}

The basic equations in vector form for an incompressible conducting micropolar fluid with thermal relaxation in the presence of both magnetic field and heat source are [3]

(1) continuity equation:

$$
\nabla \cdot \mathbf{V}=0
$$

(2) momentum equation:

$$
\begin{aligned}
\rho \frac{D \mathbf{V}}{D t}= & \rho \mathbf{f}-\nabla p+(\lambda+2 \mu+k) \nabla(\nabla \cdot \mathbf{V}) \\
& -(\mu+k) \nabla \wedge(\nabla \wedge \mathbf{V})+k(\nabla \wedge \mathbf{G})+\mathbf{J} \wedge \mathbf{B},
\end{aligned}
$$


(3) angular momentum equation:

$$
\rho j \frac{D \mathbf{G}}{D t}=(\alpha+\beta+\gamma) \nabla(\nabla \cdot \mathbf{G})+k(\nabla \wedge \mathbf{V})-\gamma \nabla \wedge(\nabla \wedge \mathbf{G})-2 k \mathbf{G}+\rho \mathbf{l}
$$

(4) generalized energy equation:

$$
\rho C_{p} \frac{D}{D t}\left(T+\tau_{0} \frac{\partial T}{\partial t}\right)=\lambda^{*} \nabla^{2} T+\left(Q+\tau_{0} \frac{\partial Q}{\partial t}\right)
$$

where $\rho$ is fluid density, $g$ acceleration due to gravity, $\mathbf{V}$ and $\mathbf{G}$ velocity and microrotation, f body force per unit mass, 1 body couple per unit mass, $p$ thermodynamic pressure, $j$ microinertia, $T$ temperature, $T_{0}$ temperature of the plane surface, $T_{\infty}$ temperature of the fluid away from the plane surface, $C_{p}$ specific heat at constant pressure, $\tau_{0}$ relaxation time, $\lambda^{\prime}$ thermal conductivity, $Q$ intensity of the applied heat source, $\alpha, \beta, \gamma, \lambda, \mu$, and $k$ material constants or viscosity coefficients, $\mathbf{B}$ the magnetic induction given by

$$
\mathbf{B}=\mu_{0} \mathbf{H}
$$

and $\mathbf{J}$ is the conduction current density given by Ohm's law

$$
\mathbf{J}=\sigma_{0}\left[\mathbf{E}+\frac{\partial \mathbf{V}}{\partial t} \times \mathbf{B}\right]
$$

where $\mathbf{H}$ is the magnetic intensity, $\mathbf{E}$ the electric intensity, $\mu_{0}$ the magnetic permeability, and $\sigma_{0}$ the electrical conductivity.

The unsteady one-dimensional vertical flow of incompressible electrically conducting micropolar fluid past an infinite plane surface is considered. The $x$-axis is taken in the vertical direction along the plate and the $y$-axis is normal to it. The velocity components of the fluid are $(u, 0,0)$ and $N$ is the local angular velocity acting in $z$ direction. A constant magnetic field with components $\left(0, H_{0}, 0\right)$ is assumed to be applied transversely to the direction of the flow. The induced electric current due to the motion of the fluid that is caused by the buoyancy forces does not distort the applied magnetic field. The previous assumption is reasonably true if the magnetic Reynolds number of the flow $\left(R_{m}=U_{0} L \sigma_{0} \mu_{e}\right)$ is assumed to be very small, which is the case in many aerodynamic applications where rather low velocities and electrical conductivities are involved. All the fluid properties are assumed constant except that the influence of the density variation with temperature is considered only in the body force term. The influence of the density variations in other terms of the momentum and the energy equations, and the variations of expansions coefficient with temperature, are considered negligible. This is the well-known Boussinesq approximation.

Given the above assumptions, we have the following.

(1) The magnetic induction has one nonvanishing component:

$$
B_{y}=\mu_{0} H_{0}=B_{0} \text { (constant) }
$$


274 Free convection flow of conducting micropolar fluid

(2) The pondermotive force $\mathbf{F}=\mathbf{J} \wedge \mathbf{B}$ has one nonvanishing component in $x$ direction:

$$
F_{x}=-\frac{\sigma_{0} B_{0}^{2} u}{\rho}
$$

(3) The following constitutive equation holds:

$$
\left(\rho_{\infty}-\rho\right)=\rho \beta_{0}\left(T-T_{\infty}\right)
$$

(4) The physical variables are functions of $y$ and $t$ only.

The system of the boundary-layer equations that govern unsteady one-dimensional free convection flow through a conducting medium of micropolar fluid in the presence of a constant magnetic field and if the body couple is absent consists of

(1) momentum equation:

$$
\frac{\partial u}{\partial t}=g \beta_{0}\left(T-T_{\infty}\right)+v(1+\Delta) \frac{\partial^{2} u}{\partial y^{2}}+\frac{k}{\rho} \frac{\partial N}{\partial y}-\frac{\sigma_{0} B_{0}^{2}}{\rho} u,
$$

(2) angular momentum equation:

$$
\rho j \frac{\partial N}{\partial t}=\gamma \frac{\partial^{2} N}{\partial y^{2}}-2 k N-k \frac{\partial u}{\partial y},
$$

(3) generalized energy equation:

$$
\rho C_{p} \frac{\partial T}{\partial t}=\lambda^{*} \frac{\partial^{2} T}{\partial y^{2}}-\rho C_{p} \tau_{0} \frac{\partial^{2} T}{\partial t^{2}}+Q+\tau_{0} \frac{\partial Q}{\partial t},
$$

where $\beta_{0}$ is the coefficient of volume expansion.

In the energy equation, terms representing viscous and Joule's dissipation are neglected as they are assumed to be very small in free convection flows [14]. Also in the energy equation, the term representing the volumetric heat source is taken as a function of the space and time variables.

We introduce the following nondimensional variables:

$$
\begin{gathered}
y^{*}=\frac{y U_{0}}{v}, \quad t^{*}=\frac{t U_{0}^{2}}{v}, \quad \tau_{0}^{*}=\frac{\tau_{0} U_{0}^{2}}{v}, \quad u^{*}=\frac{u}{U_{0}}, \\
N^{*}=\frac{v}{U_{0}^{2}} N, \quad \theta=\frac{T-T_{\infty}}{T_{0}-T_{\infty}}, \quad p_{r}=\frac{C_{p} \mu}{\lambda^{*}}, \\
G_{r}=\frac{\nu \beta g\left(T_{0}-T_{\infty}\right)}{U_{0}^{3}}, \quad Q^{*}=\frac{\nu^{2} Q}{\lambda^{*} U_{0}^{2}\left(T_{0}-T_{\infty}\right)},
\end{gathered}
$$


where $G_{r}$ is the Grashof number and $p_{r}$ the Prandtl number. Invoking the nondimensional quantities above, (2.10), (2.11), and (2.12) are reduced to the nondimensional equations, dropping the asterisks for convenience,

$$
\begin{gathered}
\frac{\partial u}{\partial t}=G_{r} \theta+(1+\Delta) \frac{\partial^{2} u}{\partial y^{2}}+\Delta \frac{\partial N}{\partial y}-M u, \\
\frac{\partial N}{\partial t}=\lambda \frac{\partial^{2} N}{\partial y^{2}}-2 \sigma N-\sigma \frac{\partial u}{\partial y}, \\
\left(\frac{\partial^{2}}{\partial y^{2}}-p_{r} \frac{\partial}{\partial t}\left(1+\tau_{0} \frac{\partial}{\partial t}\right)\right) \theta=-Q-\tau_{0} \frac{\partial Q}{\partial t} .
\end{gathered}
$$

From now on, we will consider a heat source of the form

$$
Q=Q_{0} \delta(y) H(t)
$$

where $\delta(x)$ and $H(t)$ are the Dirac delta function and Heaviside unit step function, respectively, and $Q_{0}$ is a constant.

We will also assume that the initial state of the medium is quiescent. Taking the Laplace transform, defined by the relation

$$
\bar{g}(s)=\int_{0}^{\infty} e^{-s t} g(t) d t
$$

of both sides of (2.14), we obtain that

$$
\begin{aligned}
\left((1+\Delta) \frac{\partial^{2}}{\partial y^{2}}-s-M\right) \bar{u} & =-G_{r} \bar{\theta}-\Delta \frac{\partial \bar{N}}{\partial y}, \\
\left(\frac{\partial^{2}}{\partial y^{2}}-\frac{s+2 \sigma}{\lambda}\right) \bar{N} & =\frac{\sigma}{\lambda} \frac{\partial \bar{u}}{\partial y} \\
\left(\frac{\partial^{2}}{\partial y^{2}}-p_{r} s\left(1+\tau_{0} s\right)\right) \bar{\theta} & =-Q_{0} \delta(y)\left(\frac{1+\tau_{0} s}{s}\right) .
\end{aligned}
$$

\section{State space formulation}

We will choose as state variables the temperature increment $\theta$, the velocity $u$, the angular velocity $N$, and their gradients. Equations (2.17) can be written as follows:

$$
\begin{gathered}
\frac{\partial \bar{\theta}}{\partial y}=\overline{\theta^{\prime}}, \quad \frac{\partial \bar{u}}{\partial y}=\overline{u^{\prime}}, \quad \frac{\partial \bar{N}}{\partial y}=\overline{N^{\prime}}, \\
\frac{\partial \overline{\theta^{\prime}}}{\partial y}=p s \bar{\theta}-Q_{0} \delta(y)\left(\frac{1+\tau_{0} s}{s}\right), \\
\frac{\partial \overline{u^{\prime}}}{\partial y}=a \bar{u}-b \bar{\theta}-\varepsilon \overline{N^{\prime}} \\
\frac{\partial \overline{N^{\prime}}}{\partial y}=m \bar{N}+n \overline{u^{\prime}}
\end{gathered}
$$


276 Free convection flow of conducting micropolar fluid

where $p=p_{r}\left(1+\tau_{0} s\right), a=(s+M) /(1+\Delta), b=G_{r} /(1+\Delta), \varepsilon=\Delta /(1+\Delta), m=(s+2 \sigma) / \lambda$, $n=\sigma / \lambda$.

The above equations can be written in matrix form as

$$
\frac{d \bar{f}(y, s)}{d y}=A(s) \bar{f}(y, s)+B(y, s)
$$

where

$$
\begin{gathered}
\bar{f}(y, s)=\left[\begin{array}{c}
\bar{\theta}(y, s) \\
\bar{u}(y, s) \\
\bar{N}(y, s) \\
\overline{\theta^{\prime}}(y, s) \\
\overline{u^{\prime}}(y, s) \\
\overline{N^{\prime}}(y, s)
\end{array}\right], \quad A(s)=\left[\begin{array}{cccccc}
0 & 0 & 0 & 1 & 0 & 0 \\
0 & 0 & 0 & 0 & 1 & 0 \\
0 & 0 & 0 & 0 & 0 & 1 \\
p s & 0 & 0 & 0 & 0 & 0 \\
-b & a & 0 & 0 & 0 & -\varepsilon \\
0 & 0 & m & 0 & n & 0
\end{array}\right], \\
B(y, s)=-Q_{0} \delta(y)\left(\frac{1+\tau_{0} s}{s}\right)\left[\begin{array}{l}
0 \\
0 \\
0 \\
1 \\
0 \\
0
\end{array}\right] .
\end{gathered}
$$

The formal solution of (3.5) can be expressed as

$$
\bar{f}(y, s)=\exp [A(y, s) y]\left(\bar{f}(0, s)+\int_{0}^{y} \exp [-A(s) z] B(z, s) d z\right) .
$$

In special cases when there is no heat source acting inside the medium, (3.7) simplifies to

$$
\bar{f}(y, s)=\exp [A(y, s) y] \bar{f}(0, s) .
$$

In order to solve the system (3.8), we need first to find the form of the matrix $\exp (A(s) y)$.

The characteristic equation of the matrix $A(s)$ is

$$
k^{6}-a_{11} k^{4}+a_{21} k^{2}-a_{31}=0,
$$

where

$$
\begin{aligned}
& a_{11}=m+a+p s+\varepsilon n, \\
& a_{21}=m a+p s(m+a+\varepsilon n), \\
& a_{31}=\text { maps. }
\end{aligned}
$$


The roots $\pm k_{1}, \pm k_{2}$, and $\pm k_{3}$ of (3.9) satisfy the relations

$$
\begin{gathered}
k_{1}^{2}+k_{2}^{2}+k_{3}^{2}=a_{11}, \\
k_{1}^{2} k_{2}^{2}+k_{1}^{2} k_{3}^{2}+k_{2}^{2} k_{3}^{2}=a_{21}, \\
k_{1}^{2} k_{2}^{2} k_{3}^{2}=a_{31} .
\end{gathered}
$$

One of the roots, say $k_{1}^{2}$, has a simple expression given by

$$
k_{1}^{2}=p s
$$

The other two roots $k_{2}^{2}$ and $k_{3}^{2}$ satisfy the relations

$$
\begin{aligned}
k_{2}^{2}+k_{3}^{2} & =m+a+\varepsilon n, \\
k_{2}^{2} k_{3}^{2} & =m a .
\end{aligned}
$$

The Taylor series expansion of the matrix exponential has the form

$$
\exp [A(s) y]=\sum_{n=0}^{\infty} \frac{1}{n !}[A(s) \cdot y]^{n}
$$

Using the well-known Cayley-Hamilton theorem, we can express $A^{4}$ and higher orders of the matrix $A$ in terms $I, A, A^{2}$, and $A^{3}$, where $I$ is the unit matrix of order 6 . Thus, the infinite series in (3.14) can be reduced to

$$
\begin{aligned}
\exp [A(s) y]= & a_{0}(y, s) I+a_{1}(y, s) A(s)+a_{2}(y, s) A^{2}(s)+a_{3}(y, s) A^{3}(s) \\
& +a_{4}(y, s) A^{4}+a_{5}(y, s) A^{5}
\end{aligned}
$$

where $a_{0}-a_{5}$ are some coefficients depending on $y$ and $s$. To determine these coefficients, we use the Taylor series expansions of $\exp \left( \pm k_{i} y\right), i=1,2,3,4,5,6$, together with (3.9), to obtain

$$
\begin{aligned}
& \exp \left( \pm k_{1} y\right)=a_{0} \pm a_{1} k_{1}+a_{2} k_{1}^{2} \pm a_{3} k_{1}^{3}+a_{4} k_{1}^{4} \pm a_{5} k_{1}^{5} \\
& \exp \left( \pm k_{2} y\right)=a_{0} \pm a_{1} k_{2}+a_{2} k_{2}^{2} \pm a_{3} k_{2}^{3}+a_{4} k_{2}^{4} \pm a_{5} k_{2}^{5} \\
& \exp \left( \pm k_{3} y\right)=a_{0} \pm a_{1} k_{3}+a_{2} k_{3}^{2} \pm a_{3} k_{3}^{3}+a_{4} k_{3}^{4} \pm a_{5} k_{3}^{5}
\end{aligned}
$$


278 Free convection flow of conducting micropolar fluid

The solution of the above system is given by

$$
\begin{aligned}
& a_{0}=-F\left(k_{2}^{2} k_{3}^{2} C_{1}+k_{1}^{2} k_{3}^{2} C_{2}+k_{1}^{2} k_{2}^{2} C_{3}\right), \\
& a_{1}=-F\left(k_{2}^{2} k_{3}^{2} S_{1}+k_{1}^{2} k_{3}^{2} S_{2}+k_{1}^{2} k_{2}^{2} S_{3}\right), \\
& a_{2}=F\left[\left(k_{2}^{2}+k_{3}^{2}\right) C_{1}+\left(k_{1}^{2}+k_{3}^{2}\right) C_{2}+\left(k_{1}^{2}+k_{2}^{2}\right) C_{3}\right], \\
& a_{3}=F\left[\left(k_{2}^{2}+k_{3}^{2}\right) S_{1}+\left(k_{1}^{2}+k_{3}^{2}\right) S_{2}+\left(k_{1}^{2}+k_{2}^{2}\right) S_{3}\right], \\
& a_{4}=-F\left(C_{1}+C_{2}+C_{3}\right), \\
& a_{5}=-F\left(S_{1}+S_{2}+S_{3}\right),
\end{aligned}
$$

where

$$
\begin{gathered}
F=\frac{1}{\left(k_{1}^{2}-k_{2}^{2}\right)\left(k_{2}^{2}-k_{3}^{2}\right)\left(k_{3}^{2}-k_{1}^{2}\right)}, \\
C_{1}=\left(k_{2}^{2}-k_{3}^{2}\right) \cosh \left(k_{1} y\right), \quad S_{1}=\frac{\left(k_{2}^{2}-k_{3}^{2}\right)}{k_{1}} \sinh \left(k_{1} y\right), \\
C_{2}=\left(k_{3}^{2}-k_{1}^{2}\right) \cosh \left(k_{2} y\right), \quad S_{2}=\frac{\left(k_{3}^{2}-k_{1}^{2}\right)}{k_{2}} \sinh \left(k_{2} y\right), \\
C_{3}=\left(k_{1}^{2}-k_{2}^{2}\right) \cosh \left(k_{3} y\right), \quad S_{3}=\frac{\left(k_{1}^{2}-k_{2}^{2}\right)}{k_{3}} \sinh \left(k_{3} y\right) .
\end{gathered}
$$

Substituting the expressions (3.17) into (3.15) and computing $A^{2}, A^{3}, A^{4}$, and $A^{5}$, we obtain, after some lengthy algebraic manipulations, $\exp (A(s) y)=L(y, s)=\left[L_{i j}(y, s)\right], i, j=$ $1,2,3,4,5,6$, where the entries $L_{i j}(y, s)$ are given by

$$
\begin{aligned}
& L_{11}=F\left(k_{1}^{2}-k_{2}^{2}\right)\left(k_{3}^{2}-k_{1}^{2}\right) C_{1}, \\
& L_{12}=L_{13}=0, \\
& L_{14}=F\left(k_{1}^{2}-k_{2}^{2}\right)\left(k_{3}^{2}-k_{1}^{2}\right) S_{1}, \\
& L_{15}=L_{16}=0, \\
& L_{21}=b F\left[\left(k_{1}^{2}-m\right) C_{1}+\left(k_{2}^{2}-m\right) C_{2}+\left(k_{3}^{2}-m\right) C_{3}\right], \\
& L_{22}=F\left[\left(k_{1}^{2}-k_{2}^{2}\right)\left(a-k_{3}^{2}\right) C_{2}+\left(k_{1}^{2}-k_{3}^{2}\right)\left(a-k_{2}^{2}\right) C_{3}\right], \\
& L_{23}=m \varepsilon F\left[\left(k_{2}^{2}-k_{1}^{2}\right) S_{2}+\left(k_{3}^{2}-k_{1}^{2}\right) S_{3}\right], \\
& L_{24}=b F\left[\left(k_{1}^{2}-m\right) S_{1}+\left(k_{2}^{2}-m\right) S_{2}+\left(k_{3}^{2}-m\right) S_{3}\right], \\
& L_{25}=F\left[k_{2}^{2}-m\left(k_{1}^{2}-k_{2}^{2}\right) S_{2}+\left(k_{3}^{2}-m\right)\left(k_{1}^{2}-k_{3}^{2}\right) S_{3}\right],
\end{aligned}
$$




$$
\begin{aligned}
& L_{26}=\varepsilon F\left[\left(k_{2}^{2}-k_{1}^{2}\right) C_{2}+\left(k_{3}^{2}-k_{1}^{2}\right) C_{3}\right], \\
& L_{31}=-n b F\left[k_{1}^{2} S_{1}+k_{2}^{2} S_{2}+k_{3}^{2} S_{3}\right] \text {, } \\
& L_{32}=-n a F\left[\left(k_{2}^{2}-k_{1}^{2}\right) S_{2}+\left(k_{3}^{2}-k_{1}^{2}\right) S_{3}\right] \text {, } \\
& L_{33}=F\left[\left(m-k_{3}^{2}\right)\left(k_{1}^{2}-k_{2}^{2}\right) C_{2}+\left(m-k_{2}^{2}\right)\left(k_{1}^{2}-k_{3}^{2}\right) C_{3}\right] \text {, } \\
& L_{34}=b F\left[C_{1}+C_{2}+C_{3}\right] \text {, } \\
& L_{35}=-n F\left[\left(k_{2}^{2}-k_{1}^{2}\right) C_{2}+\left(k_{3}^{2}-k_{1}^{2}\right) C_{3}\right] \text {, } \\
& L_{36}=F\left[\left(k_{2}^{2}-a\right)\left(k_{1}^{2}-k_{2}^{2}\right) S_{2}+\left(k_{3}^{2}-a\right)\left(k_{1}^{2}-k_{3}^{2}\right) S_{3}\right], \\
& L_{41}=-F k_{1}^{2}\left(k_{1}^{2}-k_{2}^{2}\right)\left(k_{1}^{2}-k_{3}^{2}\right) S_{1}, \\
& L_{42}=L_{43}=0 \text {, } \\
& L_{44}=-F\left(k_{1}^{2}-k_{2}^{2}\right)\left(k_{1}^{2}-k_{3}^{2}\right) C_{1}, \\
& L_{45}=L_{46}=0 \text {, } \\
& L_{51}=b F\left[k_{1}^{2}\left(k_{1}^{2}-m\right) S_{1}+k_{2}^{2}\left(k_{2}^{2}-m\right) S_{2}+k_{3}^{2}\left(k_{3}^{2}-m\right) S_{3}\right] \text {, } \\
& L_{52}=a F\left[\left(k_{1}^{2}-k_{2}^{2}\right)\left(k_{1}^{2}-m\right) S_{1}+\left(k_{1}^{2}-k_{3}^{2}\right)\left(k_{3}^{2}-m\right) S_{3}\right] \text {, } \\
& L_{53}=-\frac{\varepsilon m F}{n} L_{35} \\
& L_{54}=L_{21} \text {, } \\
& L_{55}=F\left[\left(k_{1}^{2}-k_{2}^{2}\right)\left(k_{2}^{2}-m\right) C_{2}+\left(k_{1}^{2}-k_{3}^{2}\right)\left(k_{3}^{2}-m\right) C_{3}\right] \text {, } \\
& L_{56}=-\varepsilon F\left[k_{2}^{2}\left(k_{1}^{2}-k_{2}^{2}\right) S_{2}+k_{3}^{2}\left(k_{1}^{2}-k_{3}^{2}\right) S_{3}\right] \text {, } \\
& L_{61}=\operatorname{bnF}\left(k_{1}^{2} C_{1}+k_{2}^{2} C_{2}+k_{3}^{2} C_{3}\right) \text {, } \\
& L_{62}=-\frac{n a}{\varepsilon} L_{26} \text {, } \\
& L_{63}=m L_{36} \text {, } \\
& L_{64}=L_{31} \text {, } \\
& L_{65}=-\frac{n}{\varepsilon} L_{56}, \\
& L_{66}=F\left[\left(k_{2}^{2}-a\right)\left(k_{1}^{2}-k_{2}^{2}\right) C_{2}+\left(k_{3}^{2}-a\right)\left(k_{1}^{2}-k_{3}^{2}\right) C_{3}\right] .
\end{aligned}
$$

It is worth mentioning here that (3.13a) and (3.13b) have been used repeatedly in order to write the above entries in the simplest possible form. We will stress here that the above expression for the matrix exponential is a formal one. In the actual physical problem the space is divided into two regions accordingly as $y \geq 0$ or $y<0$. Inside the region $0 \leq y \leq \infty$, the positive exponential terms, not bounded at infinity, must be suppressed. Thus, for $y \geq 0$ we should replace each $\sinh (k y)$ by $-(1 / 2) \exp (-k y)$ and each $\cosh (k y)$ by $(1 / 2) \exp (-k y)$. In the region $y \leq 0$ the negative exponentials are suppressed instead. 


\section{Application to infinite plane distribution of heat sources}

We will consider a conducting micropolar fluid occupying the region $y \geq 0$ whose state depends only on the space variables $y$ and time $t$. We also assume that there is a plane distribution of continuous heat sources located at the plate $y=0$.

We will now proceed to obtain the solution of the problem for the region $y \geq 0$. The solution for the other region is obtained by replacing each $y$ by $-y$.

Evaluating the integral in (3.7) using the integral properties of the Dirac delta function, we obtain

$$
\bar{f}(y, s)=L(y, s)[\bar{f}(0, s)+H(s)]
$$

where

$$
H(s)=-\frac{Q_{0}\left(1+\tau_{0} s\right)}{2 s}\left[\begin{array}{c}
\frac{1}{2 k_{1}} \\
b w \\
\frac{b w}{2 k_{1} k_{2} k_{3}\left(k_{1}+k_{2}\right)\left(k_{1}+k_{3}\right)\left(k_{2}+k_{3}\right)} \\
0 \\
\frac{1}{2} \\
0 \\
n b \\
\frac{n b}{2\left(k_{1}+k_{2}\right)\left(k_{1}+k_{3}\right)\left(k_{2}+k_{3}\right)}
\end{array}\right]
$$

and $w=k_{1} k_{2} k_{3}+m\left(k_{1}+k_{2}+k_{3}\right)$.

Equation (4.1) expresses the solution of the problem in the Laplace transform domain in terms of the vector $H(s)$ representing the applied heat source and the vector $\bar{f}(0, s)$ representing the conditions at the plane source of heat. In order to evaluate the components of this vector, we note first that due to the symmetry of the problem, the velocity component and the angular velocity component vanish at the plane source of heat, thus, at $t>0$, the boundary conditions are

$$
\begin{aligned}
u(0, t) & =0, & & \bar{u}(0, s)=0, \\
N(0, t) & =0, & & \bar{N}(0, s)=0,
\end{aligned}
$$

and the thermal condition at the plane source of heat can be obtained as follows.

Consider a short cylinder of unit base whose axis is perpendicular to the plane source of heat and whose bases lie on opposite sides of the plane. Applying Gauss's divergence theorem to this cylinder and noting that there is no heat flux through the lateral surface, we get, upon taking limits as the height tends to zero and using symmetry of 
the temperature,

$$
q(0, t)=\frac{1}{2} H(t) Q_{0}, \quad \text { or } \quad \bar{q}(0, s)=\frac{Q_{0}}{2 s},
$$

while the initial conditions are taken to be homogeneous.

We will use the generalized Fourier law of heat conduction in the nondimensional form [17], namely,

$$
q+\tau_{0} \frac{\partial q}{\partial t}=-\frac{\partial \theta}{\partial y} .
$$

Taking the Laplace transform of both sides of (4.5) and using (4.4), we get

$$
\left.\frac{\partial \bar{\theta}}{\partial y}\right|_{y=0}=-\frac{Q_{0}\left(1+\tau_{0} s\right)}{2 s}
$$

Equations (4.3) and (4.6) give three components of the vector $\bar{f}(0, s)$. To obtain the remaining three components, we substitute $y=0$ on both sides of (4.1), getting a system of linear equations whose solution gives

$$
\begin{aligned}
\bar{\theta}(0, s) & =-\frac{Q_{0}\left(1+\tau_{0} s\right)}{2 s k_{1}}, \\
\overline{u^{\prime}}(0, s) & =-\frac{Q_{0} b w\left(1+\tau_{0} s\right)}{2 s k_{1}\left(k_{1}+k_{2}\right)\left(k_{1}+k_{3}\right)\left(k_{2} k_{3}+m\right)}, \\
\overline{N^{\prime}}(0, s) & =-\frac{Q_{0} n b\left(1+\tau_{0} s\right)}{2 s k_{1}\left(k_{1}+k_{2}\right)\left(k_{1}+k_{3}\right)\left(k_{2} k_{3}+m\right)} .
\end{aligned}
$$

Inserting the values from (3.19) and (4.7) into the right-hand side of (4.1) and performing the necessary matrix operations, we obtain

$$
\begin{gathered}
\bar{\theta}(y, s)=\frac{Q_{0}\left(1+\tau_{0} s\right)}{2 s k_{1}} \exp \left(-k_{1} y\right), \\
\begin{aligned}
\bar{u}(y, s)=-\frac{Q_{0} b\left(1+\tau_{0} s\right)}{2 s \beta m}\left[\left(k_{2}-k_{3}\right) A_{1} \exp \left(-k_{1} y\right)+\left(k_{3}-k_{1}\right) A_{2} \exp \left(-k_{2} y\right)\right. \\
\left.+\left(k_{1}-k_{2}\right) A_{3} \exp \left(-k_{3} y\right)\right], \\
\bar{N}(y, s)=-\frac{Q_{0} n b\left(1+\tau_{0} s\right)}{2 s \beta}\left[\left(k_{2}-k_{3}\right)\left(k_{2}+k_{3}-w\right) \exp \left(-k_{1} y\right)+\left(k_{3}-k_{1}\right)\left(k_{3}+k_{1}-w\right)\right. \\
\left.\cdot \exp \left(-k_{2} y\right)+\left(k_{1}-k_{2}\right)\left(k_{1}+k_{2}-w\right) \exp \left(-k_{3} y\right)\right],
\end{aligned}
\end{gathered}
$$


where

$$
\begin{aligned}
\beta & =k_{1}\left(k_{1}^{2}-k_{2}^{2}\right)\left(k_{3}^{2}-k_{1}^{2}\right)\left(k_{2} b_{3}-k_{3} b_{2}\right), \\
A_{1} & =w m\left(k_{1}-k_{2}\right)-b_{2}\left[b_{3}-m k_{3}\left(k_{1}+k_{3}\right)\right], \\
A_{2} & =-b_{2}\left[b_{3}-m k_{3}\left(k_{1}+k_{3}\right)\right], \\
A_{3} & =-w m\left(k_{2}-k_{3}\right)-b_{2}\left[b_{3}-m k_{3}\left(k_{1}+k_{3}\right)\right], \\
b_{2} & =k_{2}^{2}-m, \quad b_{3}=k_{3}^{2}-m .
\end{aligned}
$$

Equation (4.8) determines completely the state of the fluid for $y \geq 0$. We mention in passing that these equations give also the solution to a semispace problem with a plane source of heat on its boundary constituting a rigid base. As mentioned before, the solution for the whole space when $y<0$ is obtained from (4.8), by taking the symmetries under considerations.

We will show that the solution obtained above can be used as a set of building blocks from which the solutions to many interesting problems can be constructed. For future reference we will write down the solution to the problem in the case when the source of heat is located in the plane $y=c$, instead of the plane $y=0$. In this case, we have

$$
\begin{aligned}
& \bar{\theta}(y, s)=\frac{Q_{0}\left(1+\tau_{0} s\right)}{2 s k_{1}} e^{ \pm k_{1}(y-c)} \\
& \bar{u}(y, s)=\frac{Q_{0} b\left(1+\tau_{0} s\right)}{2 s \beta m}\left[\left(k_{2}-k_{3}\right) A_{1} e^{ \pm k_{1}(y-c)}+\left(k_{3}-k_{1}\right) A_{2} e^{ \pm k_{2}(y-c)}\right. \\
&\left.+\left(k_{1}-k_{2}\right) A_{3} e^{ \pm k_{3}(y-c)}\right], \\
& \bar{N}(y, s)=-\frac{Q_{0} n b\left(1+\tau_{0} s\right)}{2 s \beta}\left[\left(k_{2}-k_{3}\right)\left(k_{2}+k_{3}-w\right) e^{ \pm k_{1}(y-c)}\right. \\
&+\left(k_{3}-k_{1}\right)\left(k_{1}+k_{3}-w\right) e^{ \pm k_{2}(y-c)} \\
&\left.+\left(k_{1}-k_{2}\right)\left(k_{1}+k_{2}-w\right) e^{ \pm k_{3}(y-c)}\right],
\end{aligned}
$$

where the upper (plus) sign denotes the solution in the region $y \leq c$, while the lower (minus) sign denotes the solution in the region $y>c$.

\section{Application to a semispace problem}

We will now consider the problem of a semispace with a plane source of heat located inside the medium at the position $y=c$ and subject to the following initial and boundary condition at $t \leq 0, u=N=0, T=T_{\infty}$ everywhere.

(a) The shearing stress is vanishing at the wall $(y=0)$, that is,

$$
\frac{\partial u(0, t)}{\partial y}=0 \quad \text { or } \quad \frac{\partial \bar{u}(0, s)}{\partial y}=0, \quad t>0 .
$$


(b) The microrotation is vanishing at the wall $(y=0)$. This represents the case of concentrated particle flows in which the microelements close to the wall are not able to rotate [16], that is,

$$
N(0, t)=0 \quad \text { or } \quad \bar{N}(0, s)=0, \quad t>0 .
$$

(c) The temperature is kept at a constant value $T_{\infty}$, which means that the temperature increment $\theta$ satisfies

$$
\theta(0, t)=0 \quad \text { or } \quad \bar{\theta}(0, s)=0, \quad t>0 .
$$

This problem can be solved in a manner analogous to the one outlined above, though the calculations will become quite messy. We will instead use the reflection method together with the solution obtained above for the whole space. This method was proposed by Ezzat in the context of the hydromagnetic boundary-layer theory [9].

The boundary conditions of the problem can be satisfied by locating two heat sources in an infinite space, one positive at $y=c$ and the other negative at $y=-c$. The temperature increment $\theta$ is obtained as a superposition of the temperature for both plane distributions. Thus $\theta=\theta_{1}+\theta_{2}$, where $\theta_{1}$ is the temperature due to the positive heat source, given by (4.10), and $\theta_{2}$ is the temperature due to the negative heat source and is obtained from (4.10) by replacing $c$ with $-c$ and noting that for all points of the semispace we have $y+c>0$. Thus, $\theta_{2}$ is given by

$$
\theta_{2}(y, s)=\frac{Q_{0}\left(1+\tau_{0} s\right)}{2 s k_{1}} e^{-k_{1}(y+c)} .
$$

Combining (4.10) and (5.3), we obtain

$$
\begin{array}{ll}
\bar{\theta}(y, s)=\frac{Q_{0}\left(1+\tau_{0} s\right)}{2 s k_{1}} e^{-k_{1} y} \sinh k_{1} c, & \text { for } y \geq c, \\
\bar{\theta}(y, s)=\frac{Q_{0}\left(1+\tau_{0} s\right)}{2 s k_{1}} e^{-k_{1} c} \sinh k_{1} y, & \text { for } y<c .
\end{array}
$$

Clearly, this distribution satisfies the boundary condition (5.3). We turn now to the problem of finding the distributions velocity, the induced magnetic field, and the electric field. Unfortunately, the above procedure of superposition cannot be applied to these fields as to the temperature fields. We define the scalar stream function $\Psi$ by the relation

$$
\bar{u}=\frac{\partial \bar{\Psi}}{\partial y} .
$$


Integrating (4.11) and using (5.6), we obtain the stream function due to the positive heat source at the position $y=c$ as

$$
\begin{aligned}
\bar{\Psi}=\frac{b Q_{0}\left(1+\tau_{0} s\right)}{2 s \beta m}[ & \left(k_{2}-k_{3}\right) A_{1} \frac{e^{ \pm k_{1}(y-c)}}{k_{1}}+\left(k_{3}-k_{1}\right) A_{2} \frac{e^{ \pm k_{2}(y-c)}}{k_{2}} \\
& \left.+\left(k_{1}-k_{2}\right) A_{3} \frac{e^{ \pm k_{3}(y-c)}}{k_{3}}\right],
\end{aligned}
$$

where the upper sign is valid for the region $0 \leq y<c$ and the lower sign is valid for the region $y \geq 0$. Similarly the stream function for the negative heat source at $y=-c$ is given by

$$
\begin{aligned}
\bar{\Psi}=\frac{b Q_{0}\left(1+\tau_{0} s\right)}{2 s \beta m}[ & \left(k_{2}-k_{3}\right) A_{1} \frac{e^{-k_{1}(y+c)}}{k_{1}} \\
& \left.+\left(k_{3}-k_{1}\right) A_{2} \frac{e^{-k_{2}(y+c)}}{k_{2}}+\left(k_{1}-k_{2}\right) A_{3} \frac{e^{-k_{3}(y+c)}}{k_{3}}\right] .
\end{aligned}
$$

Since $\Psi$ is a scalar field, we can use superposition to obtain the stream function for the semispace problem as

$$
\bar{\Psi}=\left\{\begin{aligned}
\frac{b Q_{0}\left(1+\tau_{0} s\right)}{s \beta m}[ & \left(k_{2}-k_{3}\right) A_{1} \frac{e^{-k_{1} y} \sinh k_{1} c}{k_{1}}+\left(k_{3}-k_{1}\right) A_{2} \frac{e^{-k_{2} y} \sinh k_{2} c}{k_{2}} \\
& \left.+\left(k_{1}-k_{2}\right) A_{3} \frac{e^{-k_{3} y} \sinh k_{3} c}{k_{3}}\right], \quad \text { for } y \geq c, \\
\frac{b Q_{0}\left(1+\tau_{0} s\right)}{s \beta m}[ & \left(k_{2}-k_{3}\right) A_{1} \frac{e^{-k_{1} c} \sinh k_{1} y}{k_{1}}+\left(k_{3}-k_{1}\right) A_{2} \frac{e^{-k_{2} c} \sinh k_{2} y}{k_{2}} \\
& \left.+\left(k_{1}-k_{2}\right) A_{3} \frac{e^{-k_{3} c} \sinh k_{3} y}{k_{3}}\right], \text { for } y<c .
\end{aligned}\right.
$$

Using (5.6) and (5.9), we obtain the velocity distribution

$$
\bar{u}=\left\{\begin{array}{c}
-\frac{b Q_{0}\left(1+\tau_{0} s\right)}{s \beta m}\left[\left(k_{2}-k_{3}\right) A_{1} e^{-k_{1} y} \sinh k_{1} c+\left(k_{3}-k_{1}\right) A_{2} e^{-k_{2} y} \sinh k_{2} c\right. \\
\left.+\left(k_{1}-k_{2}\right) A_{3} e^{-k_{3} y} \sinh k_{3} c\right], \quad \text { for } y \geq c, \\
-\frac{b Q_{0}\left(1+\tau_{0} s\right)}{s \beta m}\left[\left(k_{2}-k_{3}\right) A_{1} e^{-k_{1} c} \cosh k_{1} y+\left(k_{3}-k_{1}\right) A_{2} e^{-k_{2} c} \cosh k_{2} y\right. \\
\left.+\left(k_{1}-k_{2}\right) A_{3} e^{-k_{3} c} \cosh k_{3} y\right], \quad \text { for } y<c .
\end{array}\right.
$$


Differentiating (5.10) and using the resulting expressions together with (3.4), we obtain

$$
\bar{N}=\left\{\begin{array}{c}
-\frac{n b Q_{0}\left(1+\tau_{0} s\right)}{s \beta}[ \\
{\left[k_{2}-k_{3}\right)\left(k_{2}+k_{3}-w\right) e^{-k_{1} y} \sinh k_{1} c} \\
+\left(k_{3}-k_{1}\right)\left(k_{1}+k_{3}-w\right) e^{-k_{2} y} \sinh k_{2} c \\
\left.+\left(k_{1}-k_{2}\right)\left(k_{1}+k_{2}-w\right) e^{-k_{3} y} \sinh k_{3} c\right], \quad \text { for } y \geq c \\
-\frac{n b Q_{0}\left(1+\tau_{0} s\right)}{s \beta \quad}\left[\left(k_{2}-k_{3}\right)\left(k_{2}+k_{3}-w\right) e^{-k_{1} c} \sinh k_{1} y\right. \\
+\left(k_{3}-k_{1}\right)\left(k_{1}+k_{3}-w\right) e^{-k_{2} c} \sinh k_{2} y \\
\left.+\left(k_{1}-k_{2}\right)\left(k_{1}+k_{2}-w\right) e^{-k_{3} c} \sinh k_{3} y\right], \quad \text { for } y<c .
\end{array}\right.
$$

Clearly, $\partial \bar{u}(0, s) / \partial y=\bar{N}(0, s)=0$, in agreement with (5.1) and (5.2).

\section{Inversion of the Laplace transform}

In order to invert the Laplace transforms in (4.8) for the whole-space problem and in (5.5), (5.10), and (5.11) for the semispace problem, we adopt a numerical inversion method based on Fourier series expansion [15]. In this method the inverse $g(t)$ of the Laplace transform $\bar{g}(s)$ is approximated by the relation

$$
g_{N}(t)=\frac{e^{d t}}{t_{1}}\left[\frac{1}{2} \bar{g}(d)+\operatorname{Re}\left(\sum_{k=1}^{N^{*}} e^{i k \pi t / t_{1}} \bar{g} d+\frac{i k \pi}{t_{1}}\right)\right], \quad 0 \leq t \leq 2 t_{1},
$$

where $N^{*}$ is a sufficiently large integer representing the number of terms in the truncated infinite Fourier series. $N^{*}$ must be chosen such that

$$
\exp \left(c_{0} t\right) \operatorname{Re}\left[\bar{f}\left(c_{0}+\frac{i N^{*} \pi}{t_{1}}\right) \exp \left(\frac{i N^{*} \pi t}{t_{1}}\right)\right] \leq \varepsilon_{0}
$$

where $\varepsilon_{0}$ is a reselected small positive number that corresponds to the degree of accuracy required. The parameter $c_{0}$ is a positive free parameter that must be greater than the real parts of all singularities of $\bar{g}(s)$. The optimal choice of $c_{0}$ was obtained according to the criteria described in [15].

In order to find the temperature distribution $\theta$, we use expression (6.1) with $\theta$ and $\bar{\theta}$ replacing $g$ and $\bar{g}$, respectively. This procedure is repeated for the other functions.

\section{Numerical results}

The investigation of the effect of the magnetic field, Prandtl number, and relaxation time on the free convection of conducting micropolar fluid along an infinite nonmagnetic plane surface has been carried out in the preceding sections. The one-dimensional problem for the whole space with a plane distribution of heat sources has been studied. The 


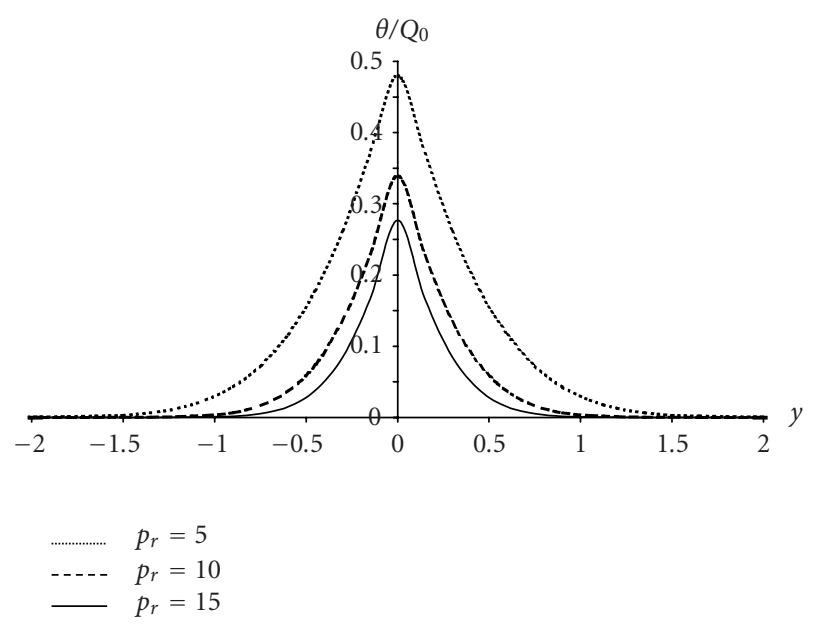

Figure 7.1. The temperature distribution for different values of $p_{r}$ for the whole-space problem.

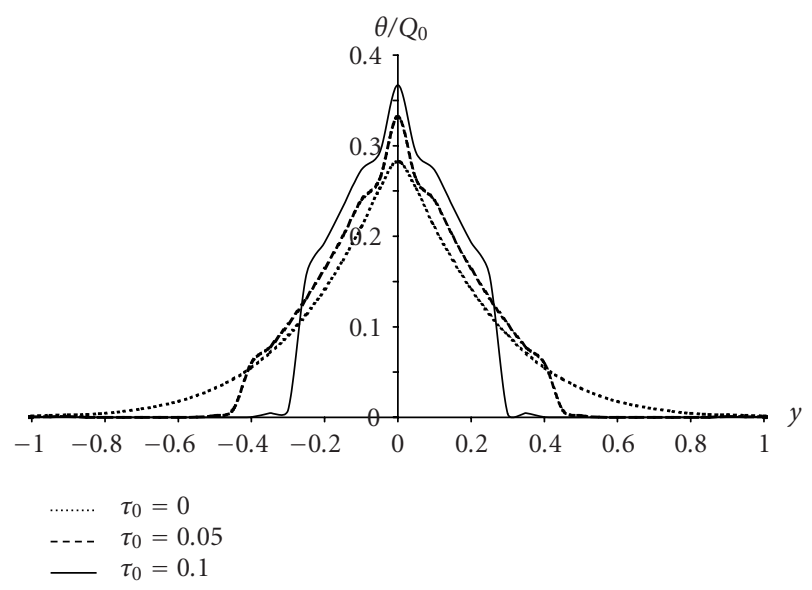

Figure 7.2. The temperature distribution for different values of $\tau_{0}$ for the whole space problem.

solutions obtained are utilized in combination with the method of images to obtain the solution for a semispace problem with a plane distribution of heat sources located inside the fluid. This enables us to represent the typical numerical results in figures for the temperature, velocity, and angular velocity for various values of the parameters. Hence we conclude with the following points.

(i) Figures 7.1, 7.2, 7.3, 7.4, 7.5, and 7.6 represent the solution for the whole-space problem while Figures 7.7, 7.8, and 7.9 represent the solution for the semispace problem. 


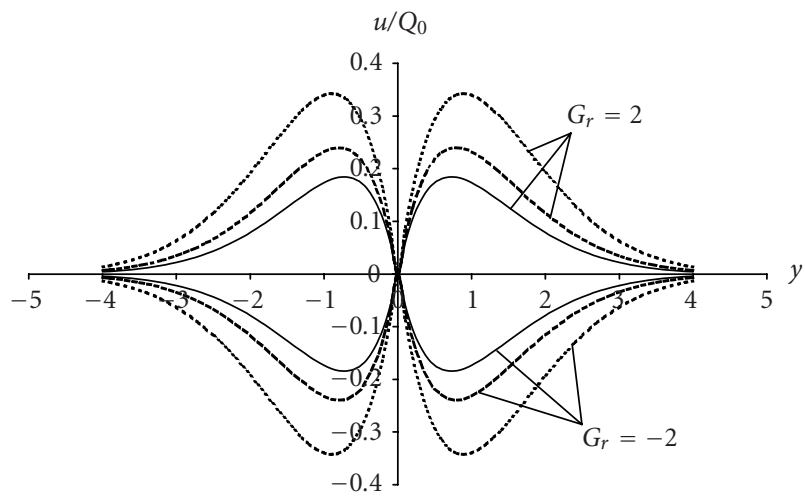

$\begin{array}{ll}\ldots \cdots \cdots & M=0 \\ --- & M=2 \\ - & M=4\end{array}$

Figure 7.3. The velocity distribution for different values of $M$ for the whole-space problem.
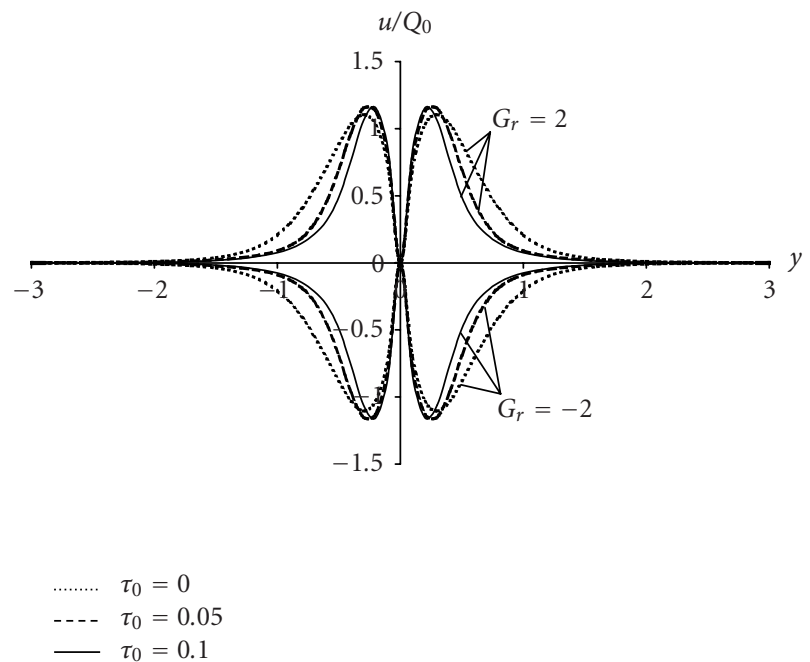

Figure 7.4. The velocity distribution for different values of $\tau_{0}$ for the whole-space problem.

(ii) In Figures 7.3, 7.4, 7.5, and 7.6 and Figures 7.8 and 7.9, we observe that the effect of cooling and heating by free convection currents occurs when $G_{r}>0$ and $G_{r}<0$ are in agreement with physical observations that cooling of the surface by free convection currents occurs for positive values of $G_{r}$ while heating corresponds to negative values of $G_{r}$. It was also noticed that the velocity and angular velocity increase with the increase of $G_{r}$. 


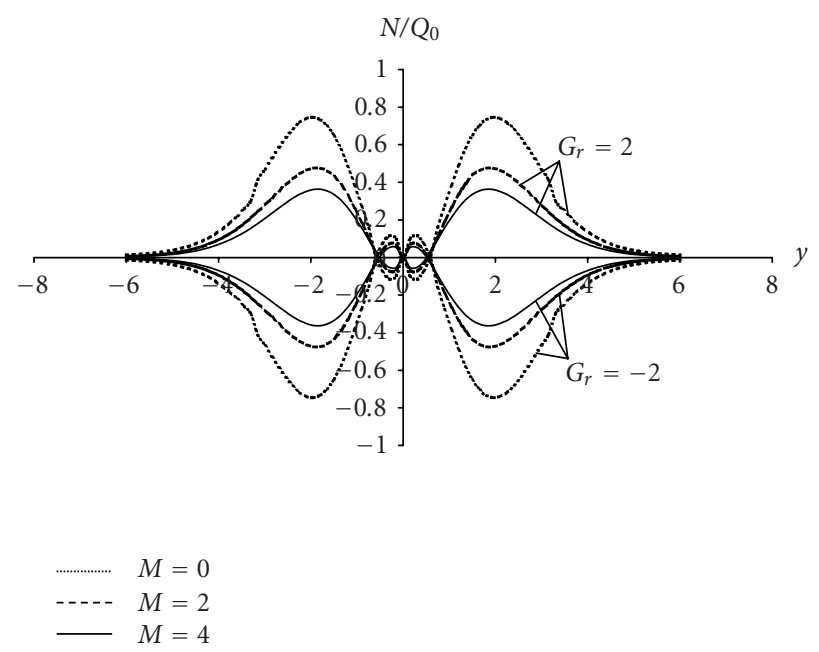

Figure 7.5. The angular velocity distribution for different values of $M$ for the whole-space problem.
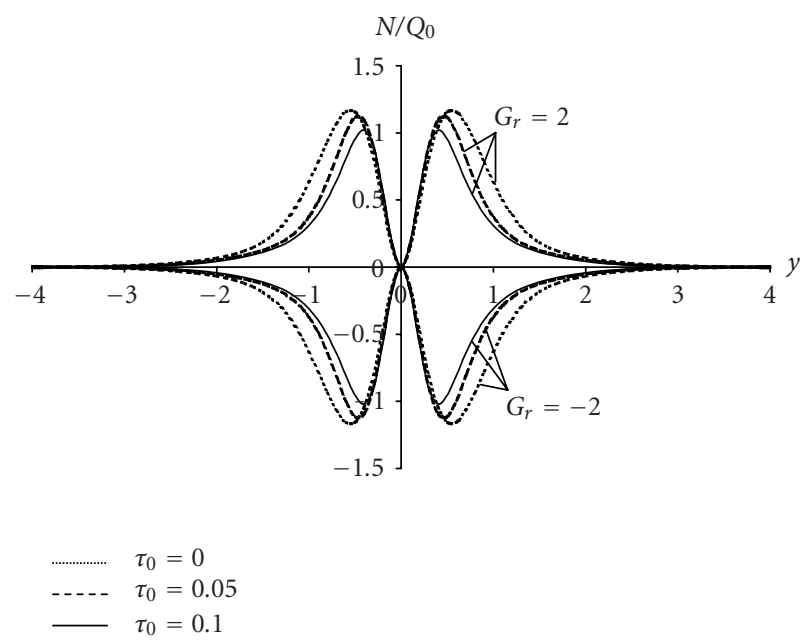

Figure 7.6. The angular velocity distribution for different values of $\tau_{0}$ for the whole-space problem.

(iii) The velocity and angular velocity profiles for cooling micropolar fluid $\left(G_{r}>0\right)$ and for heating micropolar fluid $\left(G_{r}<0\right)$ are represented in Figures 7.3, 7.4, 7.5, 7.6, 7.8, and 7.9 for different values of $M$ and $\pi_{0}$. It is observed that the increase of $M$ and $\pi_{0}$ leads to a decrease in the velocity and angular velocity.

(iv) The temperature distribution for both problems are represented graphically in Figures 7.1, 7.2, and 7.7 for different values of Prandtl number $p_{r}$ and $\tau_{0}$. It was found that the increasing of $p_{r}$ leads to a decrease of temperature, while the increase in $\tau_{0}$ leads to an increase in the temperature. 


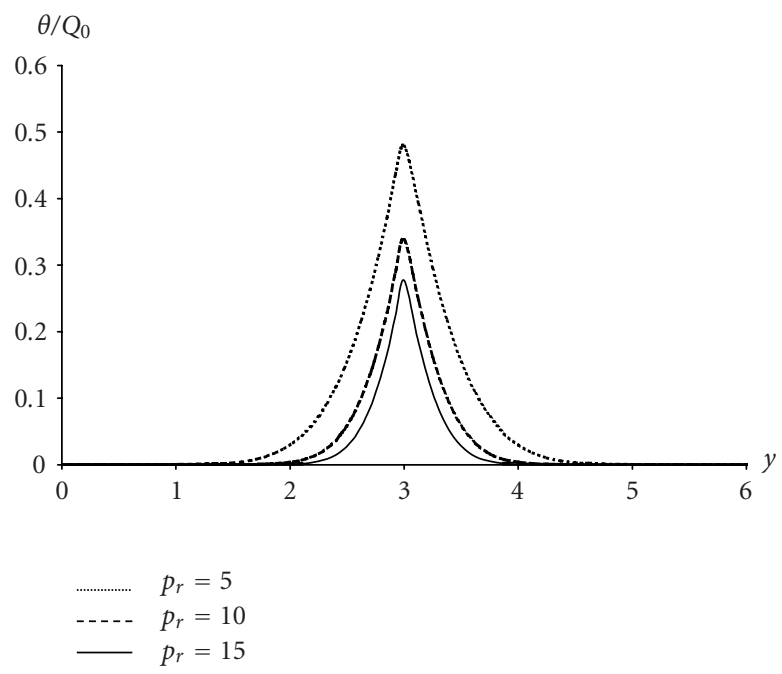

Figure 7.7. The temperature distribution for different values of $p$ for the semispace problem.
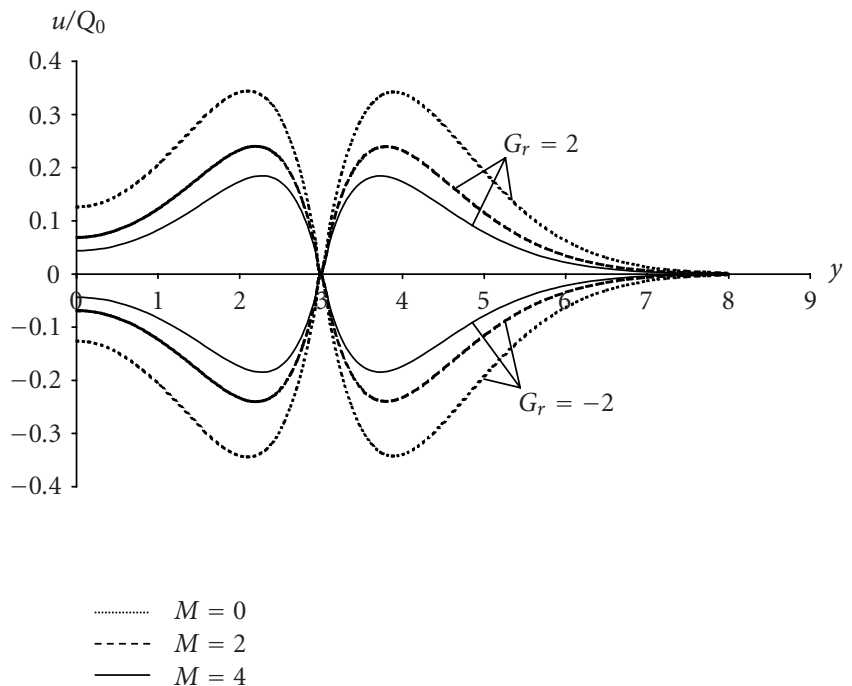

Figure 7.8. The velocity distribution for different values of $M$ for the semispace problem.

(v) The important phenomenon observed in all computations is that the solution of any of the considered functions vanishes identically outside a bounded region of space surrounding the heat source at a distance from it equal to $y^{*}(t)$. Say $y^{*}(t)$ is a particular value of $y$ depending only on the choice of $t$ and is the location of the wave front. This demonstrates clearly the difference between the solution 


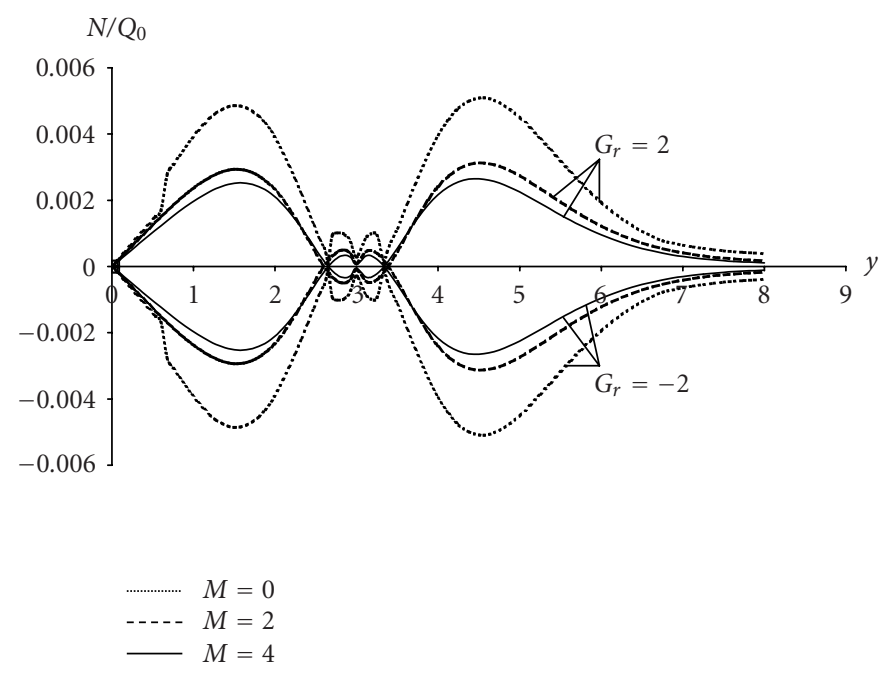

Figure 7.9. The angular velocity distribution for different values of $M$ for the semispace problem.

corresponding to using classical Fourier heat equation $\left(\tau_{0}=0.0\right)$ and that according to using the generalized Fourier case $\left(\tau_{0}=0.2\right)$. In the first and older theory, the waves propagate with infinite speeds, so the value of any of the functions is not identically zero (though it may be very small) for any large value of $y$. In non-Fourier theory the response to the thermal and mechanical effects does not reach infinity instantaneously but remains in a bounded region of space given by $0<y<y^{*}(t)$ for the semispace problem and by $\min \left(0, y^{*}(t)-c\right)<y<y+y^{*}(t)$ for the whole-space problem.

\section{Concluding remarks}

(i) A new stage in the evaluation of fluid-dynamic theory is in progress because of the increasing importance, in the processing industries and elsewhere, of materials whose flow behavior in shear cannot be characterized by Newtonian relationships. The theory of micropolar fluids can be used to explain the flow of colloidal fluids, liquid crystals, human and animal blood, and so forth.

(ii) Many metallic materials are manufactured after they have been refined sufficiently in the molten state. Therefore, it is a central problem in metallurgical chemistry to study the free convection effects on conducting liquid metal. For instance, liquid sodium $\mathrm{Na}$ $\left(100^{\circ} \mathrm{C}\right)$ and liquid potassium $\mathrm{K}\left(100^{\circ} \mathrm{C}\right)$ exhibit very small electrical resistivity $\left(\rho_{L}(\exp )\right.$ $=9.6 \times 10^{-6} \Omega \mathrm{cm}$ and $\left.\rho_{L}(\exp )=12.97 \times 10^{-6} \Omega \mathrm{cm}\right)$.

(iii) The state space approach is more general than the classical Laplace and Fourier transform techniques. Consequently, state space is applicable to all systems that can be analyzed by integral transforms in time, and is applicable to many systems for which transform theory breaks down [21]. 
(iv) Owing to the complicated nature of the governing equations for the unsteady magnetohydrodynamic micropolar flow, few attempts have been made to solve problems in this field. These attempts utilized approximate methods valid for only a specific range of some parameters. In this work, the method of direct integration by means of the matrix exponential, which is a standard approach in modern control theory and is developed in detail in many texts such as $[5,8,18]$ is introduced in the field of magnetohydrodynamic and is applied to specific problems in which the temperature, velocity, and magnetic field are coupled. This method gives exact solutions in the Laplace transform domain without any assumed restrictions on the applied magnetic field or the velocity, temperature distributions, and micropolar parameters. The same approach was used quite successfully in dealing with problems in generalized thermoelasticity theory [7].

\section{References}

[1] G. Ahmadi, Self-similar solution of incompressible micropolar boundary layer flow over a semiinfinite plate, Int. J. Engin. Sci. 14 (1976), 639-646.

[2] R. Bhargava and M. Rani, Heat transfer in micropolar boundary layer flow near a stagnation point, Int. J. Enging. Sci. 23 (1985), 1331-1335.

[3] A. C. Eringen, Theory of micropolar fluids, J. Math. Mech. 16 (1966), 1-18.

[4] Theory of thermomicrofluids, J. Math. Anal. Appl. 38 (1972), 480-496.

[5] M. A. Ezzat, State space approach to unsteady free convection flow through a porous medium, Appl. Math. Comput. 64 (1994), no. 2-3, 191-205.

[6] State space approach to unsteady two-dimensional free convection flow through a porous medium, Can. J. Phys. 1 (1994), 311-317.

[7] State space approach to generalized magneto-thermoelasticity with two relaxation times in a medium of perfect conductivity, Int. J. Eng. Sci. 35 (1997), no. 8, 741-752.

[8] , Free convection effects on perfectly conducting fluid, Int. J. Eng. Sci. 39 (2001), no. 7 , 799-819.

[9] - Free convection effects on extracellular fluid in the presence of a transverse magnetic field, Appl. Math. Comput. 151 (2004), no. 2, 455-482.

[10] M. A. Ezzat and M. I. A. Othman, Thermal instability in a rotating micropolar fluid layer subject to an electric field, Int. J. Eng. Sci. 38 (2000), no. 16, 1851-1867.

[11] M. A. Ezzat, M. I. A. Othman, and K. A. Helmy, A problem of a micropolar magnetohydrodynamic boundary-layer flow, Can. J. Phys. 77 (1999), no. 10, 813-827.

[12] R. S. Gorla, A. Mohammedan, M. Mansour, and I. Hussein, Unsteady natural convection from a heated vertical plate in micropolar fluid, Numerical Heat Transfer, Part A 28 (1995), 253-262.

[13] I. Hassanien and R. Gorla, Mixed convection in stagnation flow of micropolar fluid over vertical surfaces with uniform surface heat flux, Int. J. of Fluid Eng. Mech. 5 (1992), no. 3, 391-412.

[14] J. Holman, Heat Transfer, McGraw-Hill, Kogakusha, Tokyo, 1976.

[15] G. Honig and U. Hirdes, A method for the numerical inversion of Laplace transforms, J. Comput. Appl. Math. 10 (1984), 113-132.

[16] S. K. Jena and M. N. Mathur, Similarity solutions for laminar free convection flow of a thermomicropolar fluid past a non-isothermal vertical flat plate, Int. J. Eng. Sci. 19 (1981), 1431-1439.

[17] H. W. Lord and Y. Shulman, A generalized dynamical theory of thermoelasticity, J. Mech. Phys. Solids 15 (1967), 299-309.

[18] K. Ogata, State Space Analysis of Control Systems, Prentice-Hall, New Jersey, 1967.

[19] J. R. Peddieson and R. P. McNitt, Boundary layer for a micropolar fluid, Recent Adv. Eng. Sci. 5 (1972), 23-28. 
292 Free convection flow of conducting micropolar fluid

[20] B. C. Sakiadis, Boundary layer behavior on continuous solid surface, Am. Int. Chem. Eng. J. 7 (1961), 221-225.

[21] D. Wiberg, Schaum's Outline Series in Engineering, McGraw-Hill, New York, 1971.

Magdy A. Ezzat: Department of Mathematics, Faculty of Education, Alexandria University, El-Shatby 21625, Alexandria, Egypt

E-mail address: m_ezzat2000@yahoo.com 


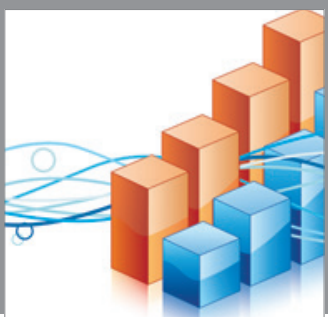

Advances in

Operations Research

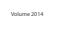

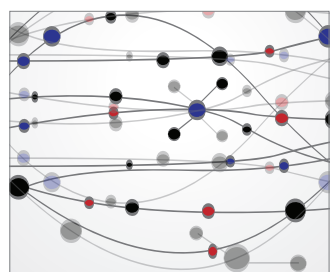

\section{The Scientific} World Journal
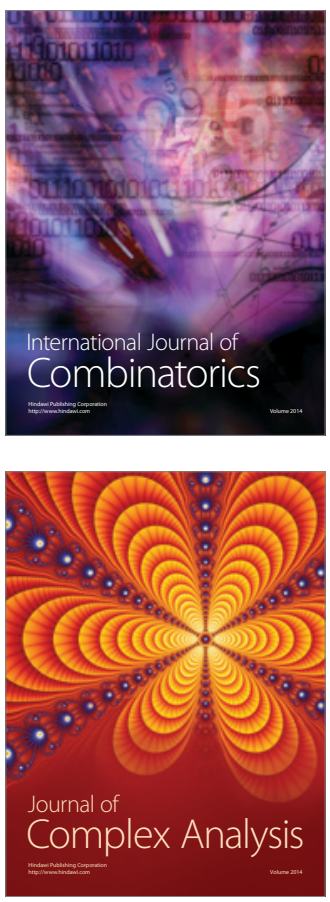

International Journal of

Mathematics and

Mathematical

Sciences
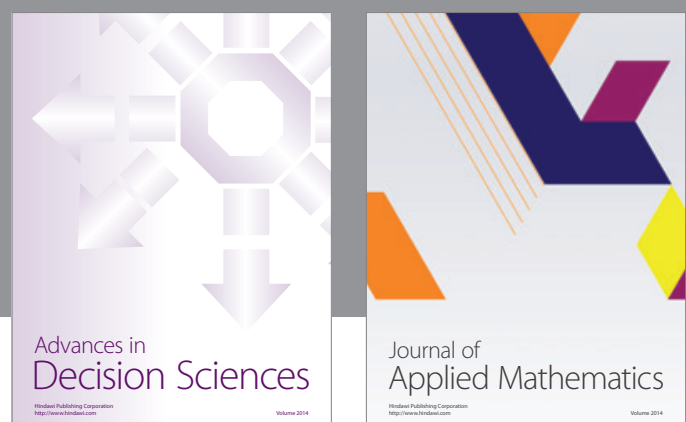

Journal of

Applied Mathematics
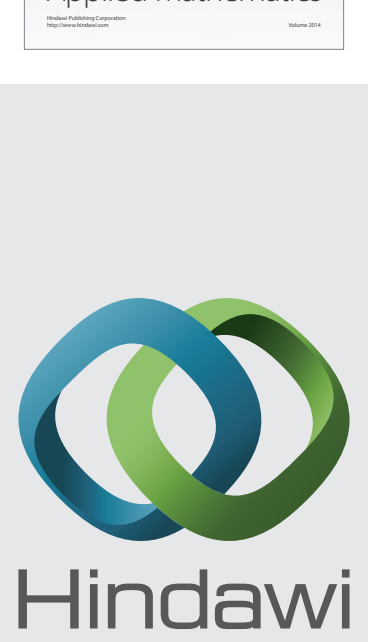

Submit your manuscripts at http://www.hindawi.com
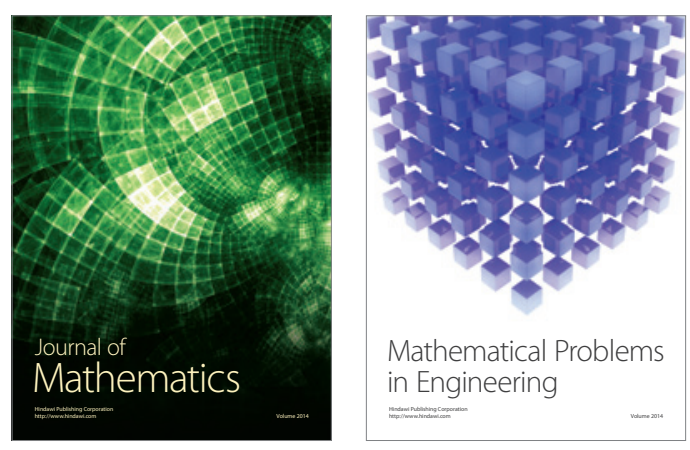

Mathematical Problems in Engineering
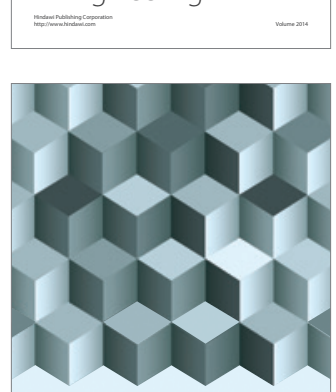

Journal of

Function Spaces
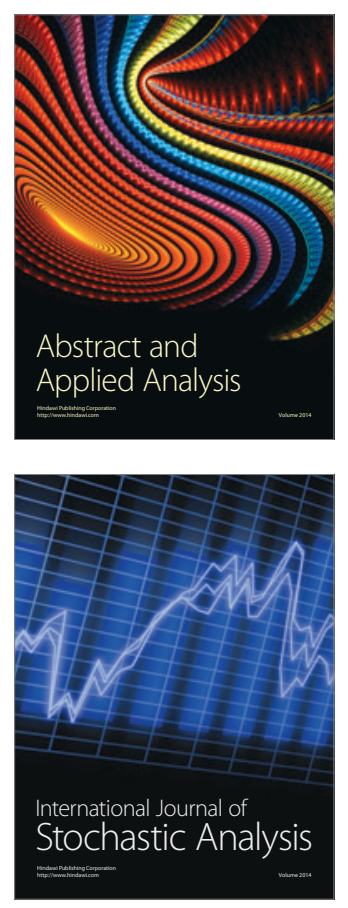

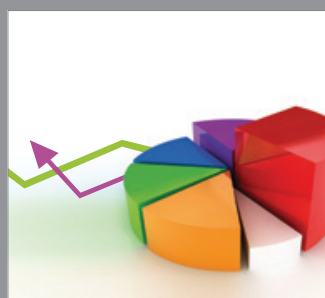

ournal of

Probability and Statistics

Promensencen
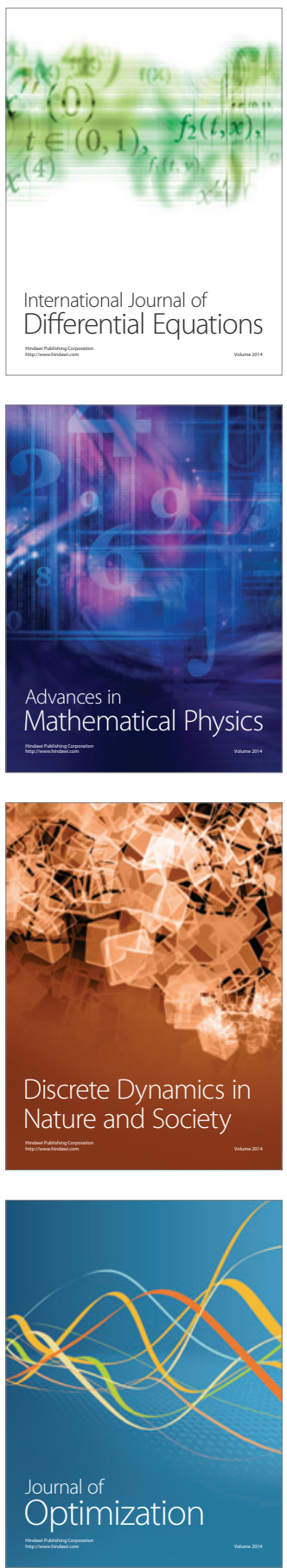